\title{
Clinical and Myopathological Characteristics of Desminopathy Caused by a Mutation in Desmin Tail Domain
}

\author{
Paul Maddison $^{\mathrm{a}}$ Maxwell S. Damian ${ }^{\mathrm{b}}$ Caroline Sewry ${ }^{\mathrm{c} d} \quad$ Catherine McGorrian $^{\mathrm{e}}$ \\ John B. Winer ${ }^{f}$ Zagaa Odgerel ${ }^{g}$ Alexey Shatunov ${ }^{g}$ Hee Suk Lee ${ }^{g}$ \\ Lev G. Goldfarb ${ }^{g}$ \\ aDepartment of Neurology, Nottingham University Hospitals, Queens Medical Centre, Nottingham,

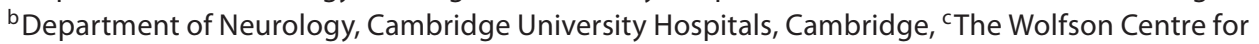 \\ Inherited Neuromuscular Diseases, Richard Jones and Agnes Hunt Orthopaedic Hospital, Oswestry, \\ dDubowitz Neuromuscular Centre, Institute of Child Health and Great Ormond Street Hospital, London, UK;

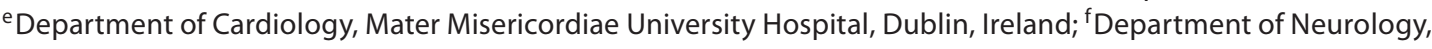 \\ Queen Elizabeth Medical Centre, University Hospitals of Birmingham, Birmingham, UK; ${ }^{9}$ Clinical Genetics Unit, \\ National Institute of Neurological Disorders and Stroke, NIH, Bethesda, Md., USA
}

\section{Key Words}

Intermediate filaments $\cdot$ Desminopathy $\cdot$ Myofibrillar

myopathy $\cdot$ Cardiomyopathy $\cdot$ Desmin gene mutations

\begin{abstract}
Background: Most of the previously described pathogenic mutations in desmin are located in highly conserved $\alpha$-helical domains that play an important role in intermediate filament assembly. The role of the C-terminus non- $\alpha$-helical 'tail' domain is much less investigated and until recently mutations in this domain have been implicated in only a few patients. The majority of reported desminopathy cases caused by the tail mutations were sporadic, creating a representation bias regarding the disease frequency and phenotypic characteristics. Methods: We performed detailed genotype-phenotype analysis of autosomal dominant desminopathy associated with tail domain mutations in a fourgeneration autosomal dominant family with 16 members affected by a progressive cardiac and/or skeletal myopathy
\end{abstract}

caused by a c.1346A $>$ C (p.Lys449Thr) mutation located in the tail domain of desmin. Results: Phenotypic features in patients with tail domain mutations are similar to those in patients with mutations localized in the $1 \mathrm{~B}$ and $2 \mathrm{~B} \alpha$-helical domains. Conclusion: We recommend that the tail domain is searched for mutations as intensely as desmin coil domains which until recently were considered to be more 'functional'.

Copyright $\odot 2012$ S. Karger AG, Basel

\section{Introduction}

Myofibrillar myopathies (MFMs) are a genetically heterogeneous group of neuromuscular disorders associated with mutations in DES, CRYAB, MYOT, ZASP, FLNC, or $B A G 3$ genes, and characterized by disintegration of myofibrils with degradation products accumulating in abnormal intracellular inclusions [1]. One of the most common variants known as desminopathy is a neuromuscular dis-

\section{KARGER}

Fax +4161306 1234 E-Mail karger@karger.ch www.karger.com
(C) 2012 S. Karger AG, Base 0014-3022/12/0685-0279\$38.00/0

Accessible online at: www.karger.com/ene
Dr. Paul Maddison

Department of Clinical Neurology

Nottingham University Hospitals, Queens Medical Centre

Nottingham NG5 1PB (UK)

Tel. +44 115924 9924, E-Mail paul.maddison@ nhs.net 
order associated with mutations in DES. More than 200 cases of desminopathy have been reported [2]. The age of disease onset, clinical/myopathological features and the rate of progression vary significantly depending on the type of inheritance and the location of the causative mutation. Typically, patients with mutations in DES present with progressive bilateral skeletal muscle weakness that starts in distal leg muscles and spreads to the proximal limb muscles and is often combined with cardiomyopathy manifested by conduction blocks, arrhythmias and chronic heart failure $[3,4]$. Some patients develop tachyarrhythmia requiring implantation of a cardioverter defibrillator [5]. Respiratory insufficiency can be a major cause of disability and death in desminopathy patients; it causes nocturnal hypoventilation with oxygen desaturation and, eventually, daytime respiratory failure [6]. Biopsies of affected skeletal and cardiac muscles show abnormal chimeric aggregates of desmin and other cytoskeletal proteins and granulofilamentous material at the ultrastructural level, among other less specific pathological features $[7,8]$.

In accordance with its function, the desmin molecule is organized into three domains: a highly conserved $\alpha$ helical core of 308 amino acid residues flanked by globular N- and C-terminal ('head' and 'tail') structures [9]. The C-terminus non-helical ('tail') domain that starts from position 416 and extends to position 470 contains $\sim 30 \%$ of $\beta$-sheet with the remainder of the domain having predominantly random structure and lacking the heptad repeat pattern. The tail is involved in the longitudinal head-to-tail tetramer assembly and control of lateral packing, stabilization and elongation of the higher order filament structures; however, the tail's major function seems to be interacting with other cytoskeletal proteins in establishing a cytoplasmic intermediate filament network [10].

Multiple DES mutations, point substitutions, an insertion, small in-frame deletions, and a larger exon-skipping deletion, have been identified in desminopathy patients. Recent filament and network assembly studies indicated that unlike the mutations located in the $\alpha$-helical segments, some tail mutations do not affect filament assembly or disrupt the filamentous network in cell cultures [11]. Nevertheless, they cause severe cardiac and skeletal myopathy [12]. This discrepancy has not been addressed or even confirmed; in particular, there were limited observations of the desminopathy phenotype in large families with DES tail mutations. The latest review [13] emphasized biochemical and biophysical consequences while the clinical/pathological characteristics of patients were sketchy: most have been published as case reports. We present here a comprehensive study of a large and clearly autosomal dominant family with a cardiac and skeletal myopathy caused by a tail domain p.Lys449Thr mutation in DES and discuss the pathological effects of previously reported tail domain mutations in light of recent experimental data.

\section{Patients and Methods}

\section{Affected Kindred}

We have identified a large family originating from Ireland and currently residing in the UK and Ireland with 16 members affected with a progressive skeletal muscle weakness and some with cardiomyopathy and respiratory failure (Pedigree, fig. 1). Clinical details on one possibly affected member (1:1) were sparse (death due to cardiac disease aged 30 years), and were therefore not included in this report. None of these patients has previously been reported. Patients were identified by local neurologists and referred to neuromuscular centres of the university hospitals of Leicester, Nottingham and Birmingham, UK, and at the Mater Misericordiae Hospital in Dublin, Ireland, for detailed studies. After obtaining informed consent, each affected family member had a neurological examination, including muscle strength assessment according to the Medical Research Council (MRC) grading scale, serum creatine kinase (CK) level assessment, electrophysiological studies that included nerve conduction tests and concentric needle electromyography (EMG), and when indicated respiratory function tests and cardiologic examination with electrocardiography and echocardiography. Fourteen patients underwent detailed cardiac investigations. Magnetic resonance images (MRI) of muscles were taken at mid-thigh and mid-calf levels in 5 patients. Time elapsed between the disease onset and the MRI examination varied from 4 to 15 years. Clinical, myopathological and molecular genetic studies were conducted under study protocols that conform to the ethical guidelines of the 1975 Declaration of Helsinki and approved by the institutional review boards of each participating institution. Informed consent was obtained in each case. Genetic testing was performed at the Clinical Neurogenetics Unit, National Institute of Neurological Disorders and Stroke, NIH, in Bethesda, Md., USA.

Muscle Biopsy

Diagnostic skeletal muscle biopsy was performed in 4 cases. Muscle was obtained from a clinically affected muscle, frozen in liquid nitrogen-cooled isopentane and processed for routine histochemical reactions and immunohistochemical analysis that included an antibody to Desmin (clone D33; Dako, Glostrup, Denmark, diluted 1:100) [14]. Samples for electron microscopy were fixed in glutaraldehyde and processed by routine methods [14].

Gene/Mutation Identification and Screening

Genomic DNA from the index case was simultaneously screened for mutations in genes known to be associated with MFM, DES, CRYAB, MYOT, ZASP, and FLNC, using previously described methodology [15]. Screening for the DES mutation in family members and unrelated controls was performed by amplification and direct sequencing of the PCR-amplified exon 8. 


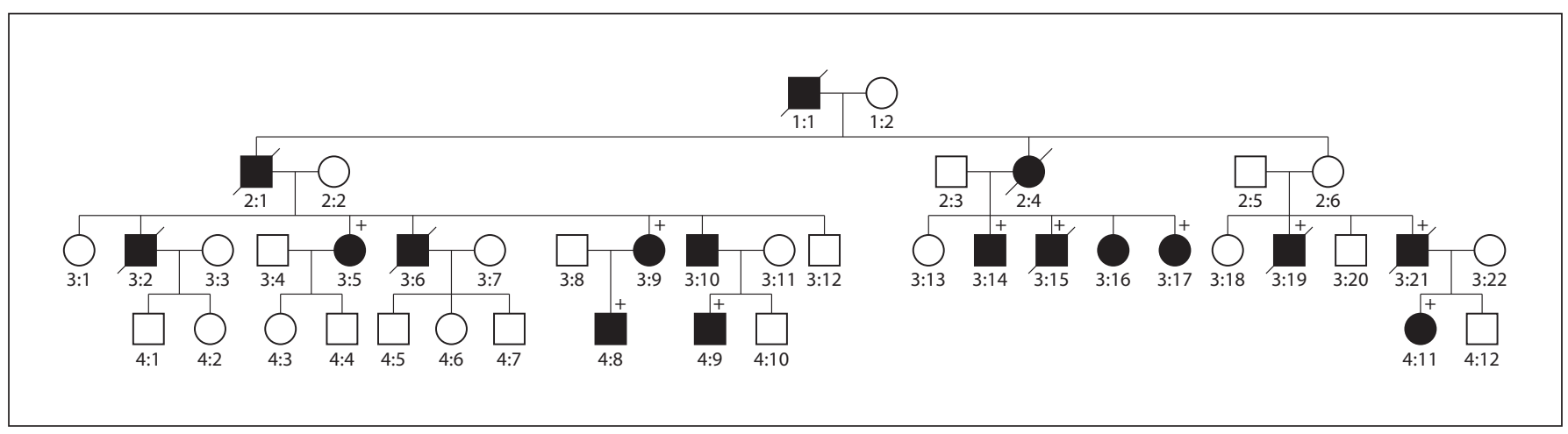

Fig. 1. Family tree of the UK-Ireland kindred segregating myopathy and cardiomyopathy associated with $D E S$ c.1346A >C (p.Lys449Thr) mutation. Filled symbols indicate affected family members; symbols with a line through indicate deceased individuals; symbols with a ' + ' indicate mutation carriers.

\section{Results}

\section{Clinical Phenotypes and Disease Outcomes}

Index Case (3:2; fig. 1)

A 38-year-old male presented with difficulty climbing stairs due to progressive proximal leg weakness. Needle EMG showed an 'irritative myopathy'; nerve conduction studies were normal, and CK was elevated at 1,044 U/1 (normal $<200 \mathrm{U} / \mathrm{l}$ ). A muscle biopsy showed infrequent eosinophilic rounded inclusions (cytoplasmic bodies) and myofibrillar disruption. The patient was started on prednisolone $30 \mathrm{mg} /$ day for suspected inflammatory myopathy, but with no positive effect. Ten years later he began to suffer intermittent dysrhythmia with atrial fibrillation and ventricular sustained polyfocal ectopic beats and was anticoagulated. Dilated cardiomyopathy was diagnosed. The patient became wheelchair bound by the age of 51, and on examination in a Muscle Disease Clinic at age 53 he had mild scapular weakness, bilateral weakness and wasting of the deltoid and triceps muscles (MRC grades 4 to $4-$ ), with relative preservation of biceps; hip flexion was antigravity, adductors were MRC grade 4-, distal leg muscles 4+. Prednisolone was withdrawn and a second muscle biopsy was performed (fig. 2). The clinical course showed slow progression of muscle weakness. The patient developed heart failure with limb oedema, and he became breathless with forced vital capacity reduced to 2 litres and falling to 1.49 litres supine, indicative of diaphragmatic weakness. He died as a result of his cardiorespiratory complications at the age of 57 years.

Desmin Tail Mutations in Desminopathy
Other Affected Family Members

Phenotypic features in other affected members of the $P W$ family are summarized in table 1 . Of 16 affected individuals, 10 were male and 6 female. The average age of disease onset was 41 within a range from 25 to 50 years. Myalgia was noted as the initial symptom in 8 patients. Progressive skeletal myopathy was present in 14 patients; muscle weakness typically started in proximal muscles of the lower or upper limbs, predominantly in the legs, and subsequently spread to distal muscles, although 2 patients developed distal lower limb weakness at onset, both in anterior and posterior compartment distal lower limb muscles. Significant facial or bulbar weakness was not observed, and spinal rigidity was not a feature in any of the kindred. In 8 patients all four limbs were eventually affected by weakness and muscle atrophy. Of 9 surviving patients, 3 are wheelchair bound. EMG studies showed motor unit potentials characteristic of myopathy and abnormal electric irritability. Muscle MRI studies performed in 5 patients revealed involvement of the calf muscle at an early stage (fig. 3a), with fatty degeneration of thigh muscles developing in a relatively selective pattern during the later course of the condition. Involvement of the semitendinosus, sartorius and gracilis muscles was prominent (fig. 3b). Ambulation with or without support was preserved for many years unless heart failure made walking impossible. Serum CK was moderately or slightly elevated in 6 of 7 tested patients.

Of 16 patients, 11 had cardiac disease, which in 6 was not disabling, but 4 developed severe cardiomyopathy (table 1). All severe phenotypes occurred in males. Of 8 males with cardiomyopathy studied by echocardiogra- 

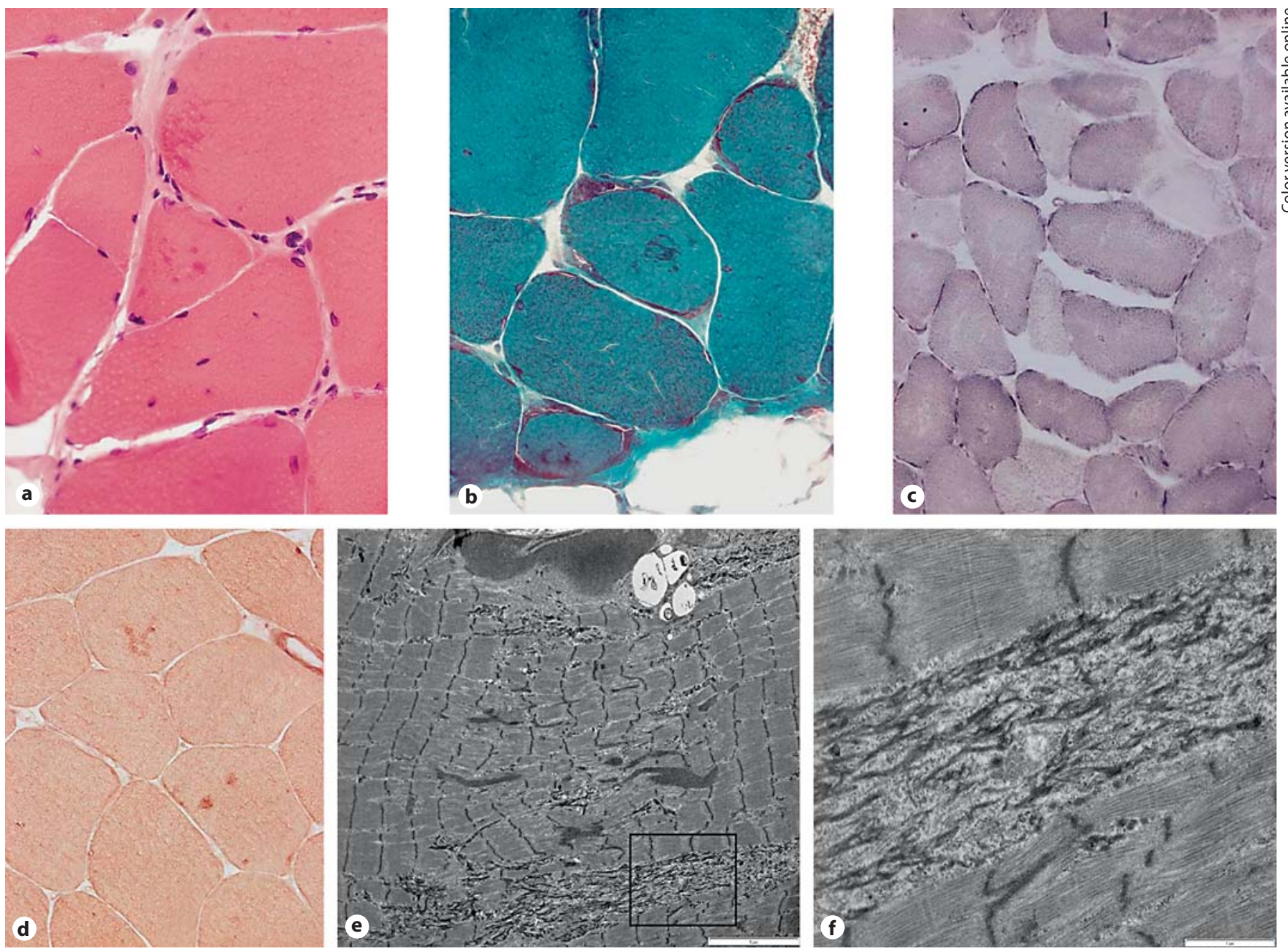

Fig. 2. Analysis of muscle biopsy samples from patients with $D E S$ c.1346A >C (p.Lys449Thr) mutation. Abnormal variation in fibre size, multiple internal nuclei, irregularly shaped eosinophilic areas with $\mathrm{HE}$ and stain (a) which are dark areas with the modified trichrome stain (b); large areas devoid of oxidative enzyme activity ('wiped out') (c), and aggregates immunoreactive for desmin (d). At electron microscopy examination, patchy electron-dense coarse granulofilmentous aggregates (e, 5 - $\mu \mathrm{m}$ resolution scale bar), most prominently near the Z-bands and beneath the sarcolemma, Zbands streaming (f, inset of $\mathbf{e}$ at $1-\mu \mathrm{m}$ resolution scale bar). phy, 1 had predominant involvement of the right ventricle, the left ventricle was affected in 3 , and both ventricles in 4 . One female had dilation of the right atrium but no ventricular dysfunction, and 1 male and 3 females had no abnormality on echocardiogram or cardiac MRI. Two patients (3:15 and 3:21) developed tachyarrhythmia requiring implantation of an ICD at the time when they already had severe cardiomyopathy and heart failure; both died within 2 years. Two patients died on the waiting list for cardiac transplantation. Cardiomyopathy progressed faster than skeletal myopathy and onset-to-death duration was on average shorter ( 9 years) than in cases with no or mild signs of heart disease (12 years). One patient (3:21) had a cardiomyopathy without signs of skeletal muscle weakness, but interestingly he complained of myalgia at the initial phase of illness. Patients with rapidly progressive disease developed heart failure on the background of dilated cardiomyopathy. Heart failure was the main cause of death, occurring at an average age of 53 years (range 48-57). Respiratory muscle weakness was detected in 6 patients, including a patient (3:16) who had neither skeletal weakness nor cardiac symptoms. Respiratory insufficiency was initially observed as exercise-induced dyspnoea and later as nocturnal hypoventilation. 

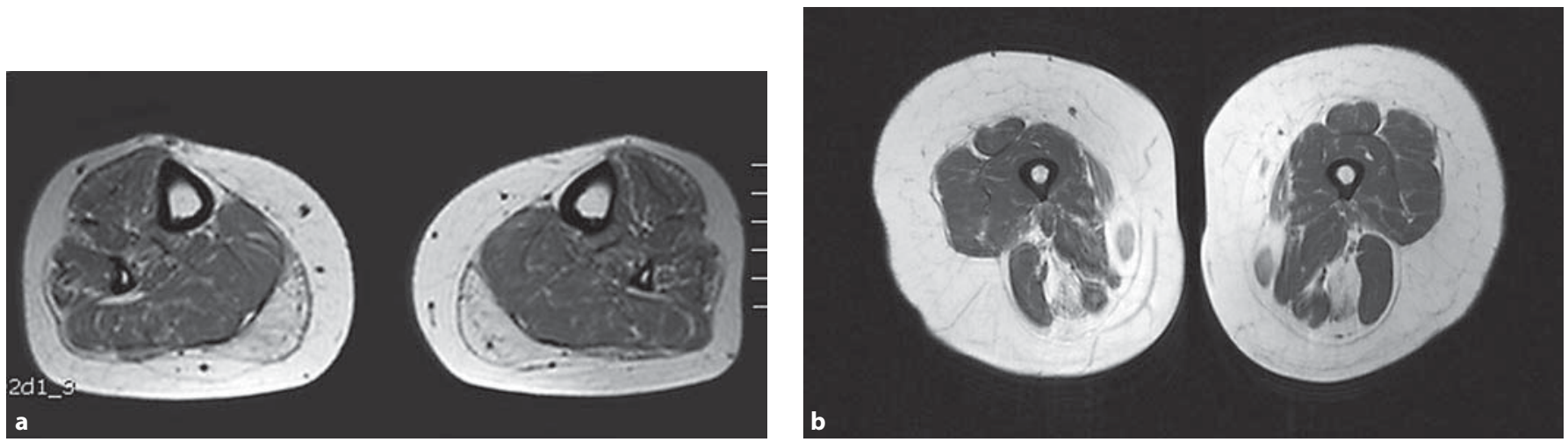

Fig. 3. Muscle MRI in patients with DES c.1346A $>$ C (p.Lys449Thr) mutation of mid-calf (a) showing selective involvement of gastrocnemius, and of mid-thigh (b) showing selective involvement of semitendinosus, gracilis and sartorius.

Table 1. Clinical manifestations of desminopathy in the affected members of the DES p.Lys449Thr family

\begin{tabular}{|c|c|c|c|c|c|c|c|}
\hline $\begin{array}{l}\text { Pedigree } \\
\text { ID }\end{array}$ & $\begin{array}{l}\text { Age of onset/ } \\
\text { current age or } \\
\text { age at death }\end{array}$ & Gender & $\begin{array}{l}\text { Initial } \\
\text { symptoms }\end{array}$ & $\begin{array}{l}\text { Muscle } \\
\text { weakness } \\
\text { distribution }\end{array}$ & $\begin{array}{l}\mathrm{CK} \\
\mathrm{IU} / 1\end{array}$ & Cardiac findings & $\begin{array}{l}\text { Respiratory } \\
\text { symptoms (FVC) }\end{array}$ \\
\hline $2: 1$ & $50 / 61^{\dagger}$ & M & $\mathrm{W}$ & UL, LL & & No data & Yes \\
\hline $2: 4$ & $? / 78^{\dagger}$ & $\mathrm{F}$ & My, W & UL, LL & & Mild failure & Unknown \\
\hline $3: 2$ & $38 / 57^{\dagger}$ & M & My, W (DLL) & PUL, LL & 1,044 & AF, RV-DCM (severe) & Yes $(2.01)$ \\
\hline $3: 5$ & $41 / 58$ & $\mathrm{~F}$ & $\mathrm{~W}(\mathrm{PLL})$ & UL, LL & & RA dilatation & No $(2.771)$ \\
\hline $3: 6$ & $? / 49^{\dagger}$ & M & $\mathrm{W}$ & $\mathrm{UL}, \mathrm{LL}$ & & LV-HCM & Yes \\
\hline 3:9 & $50 / 55$ & $\mathrm{~F}$ & My, W (PLL) & PLL & 302 & LV-HCM (mild) & No $(1.88$ l) \\
\hline $3: 10$ & $45 / 54$ & M & My, W (DLL) & UL, LL & 358 & LV-HCM (mild) & No $(2.431)$ \\
\hline $3: 14$ & $45 / 55$ & M & $\mathrm{W}$ & PLL & & Mild (ejection fraction 45\%) & No \\
\hline $3: 15$ & $? / 48^{\dagger}$ & M & My, W & PLL & & DCM (severe), ICD & Severe \\
\hline $3: 16$ & $40 / 48$ & $\mathrm{~F}$ & My & None & & Normal & Yes \\
\hline $3: 17$ & $38 / 42$ & $\mathrm{~F}$ & My & PLL, DLL & 157 & Normal & No \\
\hline $3: 19$ & $? / 52^{\dagger, *}$ & M & $\mathrm{C}$ & PLL & & DCM (severe) & Unknown \\
\hline $3: 21$ & $47 / 55^{\dagger}$ & M & My & None & 1,448 & AF, DCM (severe), ICD & Yes \\
\hline $4: 8$ & $35 / 37$ & M & $\mathrm{W}(\mathrm{PLL})$ & PUL, PLL & 319 & RBBB, HCM (mild) & No $(3.141)$ \\
\hline $4: 9$ & $34 / 35$ & M & $\mathrm{W}(\mathrm{PLL})$ & PUL, PLL & 663 & Normal & No \\
\hline $4: 11$ & $25 / 26$ & $\mathrm{~F}$ & $\mathrm{~W}$ & DLL mild & & Normal & No \\
\hline
\end{tabular}

* Died of cancer; ${ }^{\dagger}$ deceased. $\mathrm{W}=$ Weakness; My = myalgia; $\mathrm{C}=$ cardiac symptoms; $\mathrm{UL}=$ upper limbs (both proximal and distal muscles); $\mathrm{LL}=$ lower limbs (both proximal and distal muscles); $\mathrm{P}=$ proximal; $\mathrm{D}=$ distal; $\mathrm{AF}=$ atrial fibrillation; $\mathrm{RV}-\mathrm{DCM}=$ right ventricular dilated cardiomyopathy; RA = right atrium; LV-HCM = left ventricular hypertrophic cardiomyopathy; DCM = dilated cardiomyopathy (global); ICD = implanted defibrillator-cardioverter; RBBB = right bundle branch block; FVC = forced vital capacity (in litres).

\section{Myopathology}

Muscle biopsy performed in 4 affected family members at different stages of illness showed morphological hallmarks of desminopathy, including an abnormal variation in fibre size, vacuoles, increased internal nuclei that were often multiple, irregularly shaped eosinophilic areas which were dark green with the modified trichrome stain (fig. 2a, b), large areas devoid of oxidative enzyme activity ('wiped out' areas) (fig. 2c) and aggregates immunoreactive for desmin (fig. 2d) and ubiquitin. With electron microscopy examination, patchy electron-dense coarse granulofilmentous aggregates were present most promi- 
nently near the Z-bands and beneath the sarcolemma, there were notable areas of Z-band streaming and autophagic vacuoles (fig. 2e, f). A structure previously described as a cytoplasmic body was present in a single patient (not shown).

\section{Analysis of Gene Sequences and Screening for Desmin Mutation}

Analysis of DES sequences in patients 3:5, 3:9, 3:14, $3: 15,3: 17,3: 19,3: 21,4: 8,4: 9$, and $4: 11$ resulted in the identification of a heterozygous A-to-C missense mutation at mRNA position $1346(\mathrm{c} .1346 \mathrm{~A}>\mathrm{C})$ predicted to replace threonine for lysine at amino acid position 449 (p.Lys449Thr). This substitution has previously been mentioned in one of the patients with MFM at the Mayo Clinic [16] and recently in a short communication describing a single patient [17], but no detailed phenotypic information was provided. The c.1346A >C (p.Lys449Thr) mutation is located in the tail domain of the desmin molecule. Lysine at this position is strongly conserved through evolution [12]. No other mutations or unknown variations have been encountered in $D E S$, and sequencing of other MFM-associated genes, CRYAB, MYOT, ZASP, and $F L N C$ tested as a panel in the index case did not show any significant alterations. The c.1346A $>C$ (p.Lys449Thr) mutation was identified in each of the 9 molecularly analysed affected members of the $P W$ family. The mutation has not been detected by direct sequencing in 100 control individuals from the UK or 177 additional controls from mixed populations in the USA or other countries (554 chromosomes).

\section{Discussion}

We investigated an extended kinship and identified 16 patients with skeletal and cardiac manifestations of desminopathy caused by a c.1346A $>$ C (p.Lys449Thr) mutation in the tail domain of DES. Patients developed frank skeletal myopathy of predominantly limb-girdle distribution between the ages of 25 and 50 years, but this was frequently preceded by longstanding unspecific myalgia. Cardiomyopathy started later into the course of illness in 11 of 16 patients, except for one patient (3:19) with cardiomyopathy which was ultimately fatal at age 52 , without his developing severe skeletal muscle weakness. Progressive reduction of respiratory muscle strength was seen in 6 patients, one (3:16) of whom had no signs of skeletal or cardiac myopathy. Cardiomyopathy rather than respiratory failure was shown to limit life expectancy in patients with advanced disease in our pedigree. Comprehensive monitoring for cardiac disease should start early in patients with DES mutations, but we found no evidence to support prophylactic pacing or ICD implantation. These findings contrast with some of the published series of desminopathy. Significant conduction defects were less frequent in members of this family than in previously published affected families $[18,19]$.

Of 10 male patients of the $P W$ family, 4 appeared to have earlier and more severe cardiac involvement. They were diagnosed with DCM and progressed to NYHA class 4 heart failure within 10 years. Only 3 of 6 female patients had cardiac involvement, and this was much milder than in men. This correlates with data on a 10 -member family carrying DES p.Leu370Pro mutation in which the males had a higher incidence of cardiomyopathy and cardiac arrhythmia resulting in sudden death by the end of the fourth decade of life while the female patients had a slow progression of illness limited to skeletal muscles [20]. A frequently discussed controversy that the DES p.Ile451Met mutation causes DCM/no skeletal myopathy in one family [21], but this same mutation causes progressive skeletal myopathy/no cardiomyopathy in another family [22], can now be solved considering the fact that only male patients were represented in the first family and only females have been studied in the second. Further investigation needs to clarify whether this gender difference is a consistent feature of desminopathy and whether lifestyle differences play a role in its causation. Male patients with cardiomyopathy have a faster rate of progression and should be considered for cardiac transplantation earlier in the course of illness once cardiac failure develops. We have no evidence on whether medication prior to development of overt cardiomyopathy could be protective, comparable to other myopathies with predictable heart involvement [23].

Close phenotypic similarity to previously reported cases of desminopathy and the identification of a desmin mutation that was not present in 277 tested controls strengthens the diagnosis of desminopathy in the patients we studied. Nevertheless, muscle biopsy features varied significantly between the affected members of this family, and also between first and second biopsies. Desmin accumulation was not always obvious and this led to misinterpretation even in experienced laboratories. This may be due to selectivity of degenerative changes seen on muscle MRI which supports the argument for muscle imaging prior to selecting a muscle for biopsy. Nevertheless, some clinically affected muscles, such as anterior distal leg muscles, and selective thigh muscle involvement, were 
not strikingly abnormal on MRI in some of our patients. This may have been due to the few (five) patients studied with MRI, and the differences in disease duration at image acquisition. No systematic follow-up MRI scans were performed. However, it has been previously noted that individual patients with Duchenne muscular dystrophy may show unexpected relative preservation of thigh muscles on MRI despite advanced disease, including loss of ambulation [24]. Electron microscopy is a useful adjunct that allows the identification of myofibrillar disruption when light microscopy features are felt to be non-specific. Molecular diagnostic screening should not be limited to desmin exon 6 or sequences encoding the $2 \mathrm{~B}$ domain, and should always include the tail domain.

Comparative analysis of phenotypic features of the DES c.1346A >C (p.Lys449Thr) mutation with other tail domain mutations [11] indicates that the main phenotypic features are no different from the disease caused by desmin mutations occurring in the coil domains. The age of disease onset on average 33 years (range 25-50) is slightly younger than in patients with rod domain mutations; most patients with a tail mutation had a combination of cardiac and skeletal myopathy which is well known in the rod mutation-associated phenotypes. Myopathological characteristics of 4 patients in this study and previously reported patients are also identical to the findings reported in cases with mutations in coil domains, including the widespread desmin-reactive deposits and electron-dense granulofilamentous aggregates [4].
This similarity between phenotypic features of tail and non-tail mutations was difficult to explain because the tail lacks heptad repeats and the tail mutants form apparently normal filaments in transfected cells [22]. Moreover, direct assembly experiments indicated that tail mutants are able to form extended filamentous networks [11]. But lately, oscillatory shear experiments designed to characterize the mutants' mesh size of filament networks and their strain-stiffening properties revealed distinct reduction in strain stiffening due to several tail mutations, including c.1346A $>$ C (p.Lys449Thr) [25]. Expressed in combination with wild-type desmin, the p.Lys449Thr mutant no longer assembled into extended filamentous networks and instead disturbed longitudinal annealing potential of heteropolymers [25]. These findings prove that tail domain mutations affect intrafilamentous architecture and exert a dominant-negative effect on desmin assembly.

\section{Acknowledgement}

This research was supported in part by the Intramural Research Program of the National Institute of Neurological Disorders and Stroke, NIH.

\section{Disclosure Statement}

The authors have no conflicts of interest to disclose.

\section{References}

1 Selcen D, Engel AG: Myofibrillar myopathy. GeneReviews 2010. http://www.genereviews.org/.

$\checkmark 2$ Goldfarb LG, Dalakas MC: Tragedy in a heartbeat: malfunctioning desmin causes skeletal and cardiac muscle disease. J Clin Invest 2009;119:1806-1813.

3 Goebel HH, Voit T, Warlo I, Jacobs K, Johannsen U, Müller CR: Immunohistologic and electron microscopic abnormalities of desmin and dystrophin in familial cardiomyopathy and myopathy. Rev Neurol (Paris) 1994;150:452-459.

4 Dalakas MC, Park K-Y, Semino-Mora C, Lee HS, Sivakumar K, Goldfarb LG: Desmin myopathy, a skeletal myopathy with cardiomyopathy caused by mutations in the desmin gene. N Engl J Med 2000;342:770-780.
5 Luethje LG, Boennemann C, Goldfarb L, Goebel HH, Halle M: Prophylactic implantable cardioverter defibrillator placement in a sporadic desmin-related myopathy and cardiomyopathy. Pacing Clin Electrophysiol 2004;27:559-560.

6 Dagvadorj A, Goudeau B, Hilton-Jones D, Blancato JK, Shatunov A, Simon-Casteras M, Squier W, Nagle JW, Goldfarb LG, Vicart $\mathrm{P}$ : Respiratory insufficiency in desminopathy patients caused by introduction of proline residues in desmin C-terminal $\alpha$-helical segment. Muscle Nerve 2003;27:669-675.

$\checkmark 7$ Fardeau M, Godet-Guillain J, Tome FM, Collin H, Gaudeau S, Boffety C, Vernant P: Une nouvelle affection musculaire familiale, définie par l'accumulation intra-sarco-plasmique d'un matériel granulo-filamentaire dense en microscopie électronique. Rev Neurol 1978;134:411-425.

8 Goebel HH: Desmin-related neuromuscular disorders. Muscle Nerve 1995;18:1306-1320.
9 Rogers KR, Eckelt A, Nimmrich V, Janssen KP, Schliwa M, Herrmann H, Franke WW: Truncation mutagenesis of the non- $\alpha$-helical carboxyterminal tail domain of vimentin reveals contributions to cellular localization but not to filament assembly. Eur J Cell Biol 1995;66:136-150.

10 Herrmann H, Haner M, Brettel M, Müller SA, Goldie KN, Fedtke B, Lustig A, Franke WW, Aebi U: Structure and assembly properties of the intermediate filament protein vimentin: the role of its head, rod and tail domains. J Mol Biol 1996;264:933-953.

11 Bär H, Goudeau B, Walde S, Casteras-Simon M, Mücke N, Shatunov A, Goldberg YP, Clarke C, Holton JL, Eymard B, Katus HA, Fardeau M, Goldfarb L, Vicart P, Herrmann $\mathrm{H}$ : Conspicuous involvement of desmin tail mutations in diverse cardiac and skeletal myopathies. Hum Mutat 2007;28:374-386. 
12 Goldfarb LG, Olivé M, Vicart P, Goebel HH: Intermediate filament diseases: desminopathy. Adv Exp Med Biol 2008;642:131-164.

- 13 Bär H, Strelkov SV, Sjoberg G, Aebi U, Herrmann H: The biology of desmin filaments: how do mutations affect their structure, assembly, and organization? J Struct Biol 2004; 148:137-152.

14 Dubowitz V, Sewry CA: Muscle Biopsy: A Practical Approach, ed 3. New York, Elsevier, 2007.

-15 Selcen D: Myofibrillar myopathies. Curr Opin Neurol 2008;21:585-589.

-16 Selcen D, Ohno K, Engel AG: Myofibrillar myopathy: clinical, morphological and genetic studies in 63 patients. Brain 2004;127: 439-451.

-17 Albertyn C, Maguire C, Murphy RP: Myofibrillar myopathy caused by a mutation in the desmin gene: expanding the phenotype. J Neurol Neurosurg Psychiatry 2010;81:e61.
8 Arbustini E, Pasotti M, Pilotto A, Pellegrini C, Grasso M, Previtali S, Repetto A, Bellini O, Azan G, Scaffino M, Campana C, Piccolo G, Viganò M, Tavazzi L: Desmin accumulation restrictive cardiomyopathy and atrioventricular block associated with desmin gene defects. Eur J Heart Fail 2006;8:477483.

19 Vernengo L, Chourbagi O, Panuncio A, Lilienbaum A, Batonnet-Pichon S, Bruston F Rodrigues-Lima F, Mesa R, Pizzarossa C, Demay L, Richard P, Vicart P, Rodriguez MM: Desmin myopathy with severe cardiomyopathy in a Uruguayan family due to a codon deletion in a new location within the desmin $1 \mathrm{~A}$ rod domain. Neuromuscul Disord 2010;20:178-187.

20 Arias M, Pardo J, Blanco-Arias P, Sobrido MJ, Arias S, Dapena D, Carracedo A, Goldfarb LG, Navarro C: Distinct phenotypic features and gender-specific disease manifestations in a Spanish family with desmin L370P mutation. Neuromuscul Disord 2006;16: 498-503.

-21 Li D, Tapscoft T, Gonzalez O, Burch PE, Quiñones MA, Zoghbi WA, Hill R, Bachinski LL, Mann DL, Roberts R: Desmin mutation responsible for idiopathic dilated cardiomyopathy. Circulation 1999;100:461-464.
22 Dalakas MC, Dagvadorj A, Goudeau B, Park KY, Takeda K, Simon-Casteras M, Vasconcelos O, Sambuughin N, Shatunov A, Nagle JW, Sivakumar K, Vicart P, Goldfarb LG: Progressive skeletal myopathy, a phenotypic variant of desmin myopathy associated with desmin mutations. Neuromuscul Disord 2003; 13:252-258.

23 Duboc D, Meune C, Pierre B, Wahbi K, Eymard B, Toutain A, Berard C, Vaksmann G, Weber S, Bécane HM: Perindopril preventive treatment on mortality in Duchenne muscular dystrophy: 10 years' follow-up. Am Heart J 2007;154:596-602.

24 Liu GC, Jong YJ, Chiang CH, Jaw TS: Duchenne muscular dystrophy: MR grading system with functional correlation. Radiology 1993;186:475-480.

25 Bär H, Schopferer M, Sharma S Hochstein B, Mücke N, Herrmann H, Willenbacher N: Mutations in desmin's carboxy-terminal 'tail' domain severely modify filament and network mechanics. J Mol Biol 2010;397: 1188-1198. 